\title{
Association of markers of systemic inflammation, $C$ reactive protein, serum amyloid $A$, and fibrinogen, with socioeconomic status
}

\author{
P Jousilahti, V Salomaa, V Rasi, E Vahtera, T Palosuo
}

J Epidemiol Community Health 2003;57:730-733

See end of article for authors' affiliations

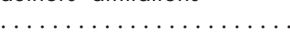

Correspondence to: Dr P Jousilahti, Nationa Public Health Institute, Department of Epidemiology and Health Promotion, Mannerheimintie 166, FIN-00300 Helsinki, Finland;

pekka.jousilahti@ktl.fi

Accepted for publication 28 February 2003

\begin{abstract}
Study objective: Systemic inflammation may play an important part in the development of cardiovascular disease. It has also been shown that socioeconomic status predicts cardiovascular events independently of established risk factors. The aim of this study was to analyse the association of three sensitive markers of systemic inflammation: $C$ reactive protein (CRP), serum amyloid A protein (SAA), and fibrinogen, with socioeconomic status.

Design: Cross sectional study.

Setting: Eastern and southern Finland.

Participants: 1503 men aged 45 to 74 years who participated in a cardiovascular risk factor survey in 1997. Based on the levels of education and family income, the men were classified to three socioeconomic groups.

Main results: Mean concentrations of CRP ( $p$ for the trend $<0.001$ ), SAA ( $p$ for the trend 0.018), and fibrinogen ( $\mathrm{p}$ for the trend $<0.001$ ) decreased substantially with increasing socioeconomic status. The trends in CRP and fibrinogen remained statistically significant after adjustment for smoking, waist to hip ratio, and prevalent longstanding diseases, and a non-significant trend was found for SAA ( $p$ for the trend 0.118 ). The inverse association between inflammation markers and socioeconomic status was particularly strong among the men below 60 years of age.

Conclusions: Systemic inflammation is a potential mediator, especially among young and middle aged men, for the association between socioeconomic status and cardiovascular disease.
\end{abstract}

$\mathrm{R}$ ecent data suggest that inflammation may play an important part in the process of atherosclerosis. ${ }^{1-6}$ An association of serological markers of bacterial and viral infections, and biochemical markers of inflammation with cardiovascular disease has been found in several studies. ${ }^{7-16}$ It has even been suggested that atherosclerosis is primarily an inflammatory disease. ${ }^{24}$

$\mathrm{C}$ reactive protein (CRP), a major acute phase protein, has been associated with the presence and severity of atherosclerosis, and has been found to predict acute cardiovascular events in prospective studies..$^{13}$ The association between plasma fibrinogen concentration, an acute phase protein and haemostatic factor, and the risk of cardiovascular disease has also been demonstrated in several studies. ${ }^{15}{ }^{16}$ An association of serum amyloid A (SAA), an acute phase protein and apolipoprotein, with cardiovascular disease has been shown mainly in cross sectional studies. ${ }^{17-19}$

Socioeconomic status predicts cardiovascular events independently of the established risk factors, such as smoking, high serum cholesterol, and hypertension..$^{20-22}$ The mechanisms behind the association between socioeconomic status and cardiovascular disease risk are not known. The association may be stronger among the middle aged than elderly people. ${ }^{21}$ Previous studies have shown an inverse association between socioeconomic status and fibrinogen but no data are available about the possible association of CRP and SAA with socioeconomic status. ${ }^{23}$

The aim of this study was to analyse the association of three sensitive markers of systemic inflammation, CRP, SAA, and plasma fibrinogen, with socioeconomic status, and furthermore, whether this association is affected by smoking, central obesity, or prevalence of longstanding diseases.

\section{METHODS}

Participants of the Finnish platelet aggregation and inflammation study (PAIS) are a sub-sample of a large population based risk factor study, which was conducted in Finland in 1997. The sub-sample included 2000 randomly selected (stratified by 10 year age group) men aged $45-74$ years from eastern and southern Finland. Of these, 1571 (79\%) participated and gave a venous blood sample. Sixty eight men were excluded because of missing data on smoking, socioeconomic status, or waist to hip ratio. Thus, 1503 men were included in these analyses. Subjects without data on CRP, SAA, and fibrinogen were excluded from the analyses where these variables were required. The study was approved by the ethical committee of the National Public Health Institute and the subjects gave informed consent.

Data on smoking, education, family income, and occurrence of longstanding diseases were obtained from a self administered questionnaire, which was sent to the participants in advance. Based on the responses, the men were classified as current smokers, ex-smokers, and never smokers. Current smokers were further classified into three groups based on the amount of smoking (1-10, 11-20, and over 20 cigarettes daily). Years of education and total family income were used to classify participants into socioeconomic classes. To adjust for changes in educational system over time (extension of basic education system and increase of average school years), the number of years of schooling were divided into tertiles separately for each birth year, and the birth cohort specific distribution was used as the indicator of socioeconomic status. Total family income was adjusted for the family size and divided into tertiles. Men in the

Abbreviations: CRP, $C$ reactive protein; $S A A$, serum amyloid A protein 
Table 1 Mean age and waist to hip ratio and prevalence of smoking and longstanding diseases by socioeconomic status

\begin{tabular}{llll}
\hline & \multicolumn{3}{l}{ Socioeconomic status } \\
\cline { 2 - 4 } & $\begin{array}{l}\text { Low } \\
(\mathrm{n}=273)\end{array}$ & $\begin{array}{l}\text { Middle } \\
(\mathrm{n}=897)\end{array}$ & $\begin{array}{l}\text { High } \\
(\mathrm{n}=333)\end{array}$ \\
\hline Age (y) & 64.8 & 61.6 & 61.0 \\
Current smokers (\%) & 34.1 & 21.5 & 21.3 \\
Ex-smokers & 36.3 & 43.2 & 39.3 \\
Waist to hip ratio & 0.96 & 0.95 & 0.94 \\
Prevalence of longstnding diseases & & & \\
Coronary heart disease (\%) & 28.6 & 20.0 & 12.9 \\
Stroke (\%) & 1.8 & 4.7 & 3.0 \\
Diabetes (\%) & 11.0 & 6.6 & 6.3 \\
Chronic bronchitis (\%) & 6.2 & 8.1 & 8.1 \\
Asthma (\%) & 9.5 & 7.8 & 5.4 \\
Cancer (\%) & 2.6 & 2.9 & 2.1 \\
Rheumatoid arthritis (\%) & 3.3 & 2.3 & 3.3 \\
Other joint disease (\%) & 10.6 & 12.2 & 7.5 \\
Urinary tract infection (\%) & 2.9 & 2.5 & 1.5 \\
Dental problems (\%) & 7.0 & 8.5 & 6.9 \\
\hline & & & \\
\hline
\end{tabular}

lowest tertile both in education and family income were classified into the low socioeconomic class, and men in the highest tertile in both indicators were classified into the high socioeconomic class. Men in the second tertile of both indicators, or with a combination of low, middle and high tertiles, were classified into the middle class. If data were missing on either indicator, socioeconomic status was assessed on the basis of the available indicator.

Data on the occurrence of coronary heart disease (angina pectoris or the history of acute myocardial infarction), past history of stroke and cancer, prevalence of rheumatoid arthritis and other joint disease, diabetes, asthma, chronic bronchitis, and chronic urinary tract infection were obtained from the questionnaire. In addition, data on the occurrence of coronary heart disease, diabetes, asthma, and rheumatoid arthritis were complemented by record linkage of the data obtained from the National Social Insurance Institution's register on persons entitled to special reimbursement for drugs. To receive the special reimbursement, the diagnosis is usually assigned by a specialist in internal medicine or other relevant specialist, and the diagnosis needs to be based on objective findings. The statements documenting these findings are then reviewed and accepted by the expert physicians of the Social Insurance Institution. Dental problems were assessed by asking whether the person had suffered from tooth pain in the past year. At the study site specially trained research nurses checked that the questionnaires were fully completed and measured waist and hip circumferences. Waist to hip ratio was used as an indicator for central obesity. ${ }^{24}$

After the measurements, a venous blood sample was obtained and centrifuged within one hour. Plasma for fibrinogen determination was frozen immediately in a dry ice/ ethanol mixture, and then stored at $-70 \mathrm{C}^{\circ}$ until laboratory analyses. Serum samples for CRP and SAA determination were transported to the central laboratory at room temperature and were stored at $-70 \mathrm{C}^{\circ}$ until the analyses. For those participants who were not able to come to the study site, a home visit with restricted data and sample collection was made. For technical reasons, plasma samples for fibrinogen determination were not taken at home visits.

CRP conentrations in serum samples were analysed in duplicate using a sandwich enzyme immunoassay kit (UC CRP ELISA, Eucardio Laboratory, San Diego, CA, USA) with a limit of detection of $350 \mathrm{ng} / \mathrm{ml}$. The average intra-assay and inter-assay precisions were $6.5 \%$ and $9 \%$, respectively. Values of SAA were analysed in duplicate using a sandwich enzyme
Table 2 Means* of $C$ reactive protein (CRP), serum amyloid $A$ protein (SAA), and fibrinogen by socioeconomic status

\begin{tabular}{llllll}
\hline & \multicolumn{5}{l}{ Socioeconomic status } \\
\cline { 3 - 6 } & & Low & Middle & High & p Value† \\
\hline CRP (mg/l) & Model 1 $\ddagger$ & 2.32 & 1.90 & 1.52 & $<0.001$ \\
& Model 2 $\ddagger$ & 2.13 & 1.90 & 1.62 & 0.016 \\
SAA (jg/l) & Model 3 & 2.11 & 1.91 & 1.63 & 0.022 \\
& Model 1 & 5.85 & 4.95 & 4.74 & 0.018 \\
& Model 2 & 5.75 & 4.95 & 4.88 & 0.085 \\
Fibrinogen (g/I) 3 & 5.64 & 4.95 & 4.91 & 0.118 \\
& Model 1 & 4.09 & 3.83 & 3.62 & $<0.001$ \\
& Model 2 & 4.02 & 3.83 & 3.65 & $<0.001$ \\
& Model 3 & 4.01 & 3.83 & 3.66 & $<0.001$ \\
\hline
\end{tabular}

* Geometric means of CRP and SAA, and arithmetic means of fibrinogen. $\uparrow p$ for the trend. $¥$ Model 1, adjusted for age; Model 2, adjusted for age, smoking, and waist to hip ratio; Model 3, adjusted for age, smoking, waist to hip ratio, and prevalent longstanding diseases. $p$ Values for the other factors in the model: age; $<0.001$ $0.010,<0.001$, current smoking; $<0.001,0.010,<0.001$, previous smoking; $0.150,0.604,0.221$, waist to hip ratio; $<0.001,<0.001$, $<0.001$, coronary heart disease; $0.006,0.211,0.199$, stroke; $0.218,0.143,0.117$, diabetes; $0.415,0.217,0.597$, chronic bronchitis; $0.080,0.793,0.040$, asthma; $0.732,0.048,0.078$, cancer; $0.352,0.091,0.432$, rheumatoid arthritis; $<0.001$, $<0.001,<0.001$, other joint disease; $0.957,0.652,0.913$, urinary tract infection; $0.229,0.208,0.831$ and dental problems; 0.999 , $0.404,0.641$, for CRP, SAA, and fibrinogen, respectively.

immunoassay kit (BioSource Cytoscreen human SAA, Camarillo, CA, USA) with a limit of detection of $5 \mathrm{ng} / \mathrm{ml}$. The average intra-assay and inter-assay precisions were $5 \%$ and $8 \%$, respectively. Fibrinogen was measured with the Clauss method $^{25}$ using IL Test Fibrinogen-C kit and a single lot of IL Test Calibration plasma. The measurements were carried out with ACL 300R equipment (Instrumentation Laboratory, Milan, Italy). The intra-assay and inter-assay precisions were $2.4 \%$ and $3.0 \%$, respectively. The means, standard deviations (SD), and median values of inflammation markers were: 4.26 (SD 9.35) and 1.60 for CRP, 10.77 (SD 42.97) and 4.60 for SAA, and 3.82 (SD 0.80) and 3.69 for fibrinogen.

The association of CRP, SAA, and fibrinogen with socioeconomic status was analysed by testing the significance of the trends over the three socioeconomic classes using general linear models. Because of the skewness of distributions, geometric means of CRP and SAA are presented and the tests were performed using log transformed values of these variables. The analyses were first adjusted for age only, and then, further for smoking, waist to hip ratio and prevalence of longstanding diseases. In the analyses, age and waist to hip ratio were included in the model as continuous variables, smoking was included as five dummy variables (based on the smoking status, and amount of smoking among current smokers) and the occurrence of lonstanding diseases was included as separate dummy variables. The combined effect and interactions of education and income level on the inflammation markers were also analysed.

\section{RESULTS}

Men with low socioeconomic status were somewhat older and they were more often smokers than men in the middle and high socioeconomic classes (table 1). Waist to hip ratio decreased slightly with increasing socioeconomic status. The prevalence of coronary heart disease, diabetes, asthma, and chronic urinary tract infection decreased with increasing socioeconomic status. As expected, the inflammation markers were closely related to each other. The age adjusted correlation coefficients were $0.47(\mathrm{p}<0.001)$ for CRP and SAA, 0.59 $(p<0.001)$ for CRP and fibrinogen, and $0.37(p<0.001)$ for SAA and fibrinogen. 
Table 3 Means* of $C$ reactive protein (CRP), serum amyloid $A$ protein (SAA), and fibrinogen by education and income levelt

\begin{tabular}{lllll}
\hline & & \multicolumn{3}{l}{ Income level } \\
\cline { 3 - 5 } & Education & Low & Middle & High \\
\hline CRP (mg/l) & Low/middle & 2.33 & 1.92 & 1.83 \\
& High & 1.90 & 1.69 & 1.48 \\
SAA ( $\mu \mathrm{g} / \mathrm{l})$ & Low/middle & 5.77 & 5.25 & 4.42 \\
& High & 5.25 & 4.39 & 4.70 \\
Fibrinogen (g/l) & Low/middle & 4.06 & 3.80 & 3.77 \\
& High & 4.00 & 3.76 & 3.59 \\
\hline
\end{tabular}

* Geometric means of CRP and SAA, and arithmetic means of fibrinogen, adjusted for age, smoking, waist to hip ratio, and prevalent longstanding diseases. IInteractions between education and income level: CRP, $p=0.912 ; \mathrm{SAA}, p=0.268$, and fibrinogen, $\mathrm{p}=0.198$.

An inverse association was found between the inflammation markers and socioeconomic status (table 2). The age adjusted levels of CRP, SAA, and fibrinogen among the men of low, middle, and high socioeconomic classes were $2.32,1.90$, and 1.52 $\mathrm{mg} / \mathrm{l}(\mathrm{p}<0.001), 5.85,4.95$, and $4.74 \mu \mathrm{g} / \mathrm{l}(\mathrm{p}=0.018)$, and 4.09, 3.83 , and $3.62 \mathrm{~g} / \mathrm{l}(\mathrm{p}<0.001)$, respectively. The trends in CRP and fibrinogen remained statistically significant also after adjustment for smoking, waist to hip ratio, and longstanding diseases. A non-significant decreasing trend was found for SAA $(p=0.118)$. Education and income level were also separately inversely associated with the inflammation markers ( $p$ values for the trend, adjusted for age, smoking, waist to hip ratio, and the occurrence of longstanding conditions: CRP, 0.024 and 0.047; SAA, 0.237 and 0.053; fibrinogen, $<0.001$ and $<0.001$, for education and income, respectively).

The joint effects of education and income level on the inflammation markers are shown in table 3. Because the effect of education was most prominent between the middle and high levels, the low and middle levels are combined. The inflammation markers decreased with increasing education at each income level, and with increasing income at each level of education, except SAA among high income men. None of the interactions between education and income were statistically significant.

In the analyses by age group, among men below 60 years of age, all three inflammation markers had an inverse association (adjusted for age, smoking, waist to hip ratio, and the prevalence of longstanding disease) with socioeconomic status ( $p$ values for the trend: CRP, $p=0.017$; SAA, $p=0.032$; fibrinogen, $p<0.001$ ) (table 4 ). In the older age group, among the men from 60 to 74 years, a statistically significant inverse association was found only between fibrinogen and socioeconomic status ( $p$ values for the trend: CRP, $p=0.267$; SAA, $\mathrm{p}=0.842$; fibrinogen, $\mathrm{p}=0.005)$. However, the interactions between age group and socioeconomic status were not significant (CRP, $p=0.247$; SAA, $p=0.192$; fibrinogen, 0.169).

\section{DISCUSSION}

All of the analysed acute phase markers, CRP, SAA, and plasma fibrinogen, were associated with socioeconomic status in this study population consisting of middle aged and elderly men. The association between inflammation markers and socioeconomic status was partly mediated through smoking, central obesity, and prevalent longstanding diseases. However, the decreasing trends of inflammation markers with increasing socioeconomic status remained significant for CRP and fibrinogen, and a non-significant trend was found for SAA, also after adjustment for smoking, waist to hip ratio, and longstanding disease.

Among the men below 60 years of age, all three markers of inflammation had a significant inverse association with socioeconomic status. The results are in accordance with the previ-
Table 4 Means* of $C$ reactive protein (CRP), serum amyloid $A$ protein, (SAA) and fibrinogen by socioeconomic status and age group

\begin{tabular}{|c|c|c|c|c|c|}
\hline & & Socio & onomic s & atus & \\
\hline & & Low & Middle & High & $\mathrm{p}$ Value \\
\hline CRP (mg/l) & $\begin{array}{l}<60 \text { years } \\
60-74 \\
\text { years }\end{array}$ & $\begin{array}{l}2.18 \\
2.15\end{array}$ & $\begin{array}{l}1.58 \\
2.13\end{array}$ & $\begin{array}{l}1.31 \\
1.83\end{array}$ & $\begin{array}{l}0.017 \\
0.267\end{array}$ \\
\hline SAA ( $\mu g / l)$ & $\begin{array}{l}<60 \text { years } \\
60-74 \\
\text { years }\end{array}$ & $\begin{array}{l}6.25 \\
5.54\end{array}$ & $\begin{array}{l}4.45 \\
5.32\end{array}$ & $\begin{array}{l}4.24 \\
5.28\end{array}$ & $\begin{array}{l}0.032 \\
0.842\end{array}$ \\
\hline Fibrinogen (g/l) & $\begin{array}{l}<60 \text { years } \\
60-74 \\
\text { years }\end{array}$ & $\begin{array}{l}4.03 \\
4.05\end{array}$ & $\begin{array}{l}3.66 \\
3.94\end{array}$ & $\begin{array}{l}3.45 \\
3.78\end{array}$ & $\begin{array}{l}<0.001 \\
0.005\end{array}$ \\
\hline
\end{tabular}

* Geometric means of CRP and SAA, and arithmetic means of fibrinogen, adjusted for age, smoking, waist to hip ratio, and prevalent longstanding diseases. $t p$ for trend.

ous findings that socioeconomic differences in cardiovascular disease morbidity and mortality are most prominent among young subjects, and diminish with increasing age. ${ }^{21}$ In older age, the socioeconomic differences in the levels of inflammation markers may also be masked by the common occurrence of longstanding diseases in all socioeconomic groups.

The association of CRP and fibrinogen levels with coronary heart disease risk has been shown both in cross sectional and prospective studies. ${ }^{13-16}{ }^{26-29}$ The evidence for a causal role of CRP in the pathophysiology of coronary heart disease is, however, inconclusive. It has been hypothesised that CRP may directly interact with atherosclerotic vessels or ischaemic myocardium by activation of the complement system and thereby promote inflammation and thrombosis. ${ }^{30}$ It has been shown also that CRP adds to the predictive value of lipid parameters in determining the risk of myocardial infarction. ${ }^{31}{ }^{32}$ Fibrinogen increases the risk of an acute coronary event through its prothrombotic and rheological effects, and may also play a part in atherosclerosis formation. ${ }^{15}{ }^{16}$ The question whether SAA is associated with increased coronary heart disease risk is new and its pathogenetic role in the disease development is still open. ${ }^{17-19} 33$

In most populations, subjects with low socioeconomic status have considerably higher cardiovascular disease risk compared with subjects with high socioeconomic status. ${ }^{20-22}$ Part of the association between socioeconomic status and the risk of cardiovascular disease is explained by the known risk factors, such as differences in smoking prevalence, blood pressure, and serum lipid concentrations. ${ }^{34}$ However, even though adjustment for the established risk factors weakens the association between socioeconomic status and cardiovascular disease risk, other factors are also involved.

Smoking, obesity, and prevalence of longstanding diseases differ between socioeconomic groups. All these factors affect also the levels of sensitive inflammation markers. ${ }^{35-37}$ The production of acute phase proteins is regulated by cytokines. ${ }^{38}$ At least interleukin 1 , interleukin 6 , and tumour necrosis factor $\alpha$ play a part in the regulation of CRP, SAA, and fibrinogen formation in the liver. ${ }^{39}$ During inflammation, cytokines are produced by inflammatory cells in the damaged tissue. ${ }^{40}$ Cytokines are also produced in adipose tissue, and therefore obesity is a potential confounding factor in the association between acute phase proteins and cardiovascular disease risk. ${ }^{35}{ }^{41}$ It has been shown that even exposure to environmental tobacco smoke increases plasma fibrinogen concentrations markedly. ${ }^{42}$

Our study provides indirect evidence that longstanding systemic low level inflammation plays a part in the association between low socioeconomic status and high cardiovascular disease risk. Systemic inflammation is also a biologically 
plausible mediator between socioeconomic status, a social phenomenon, and the pathophysiological changes behind clinical cardiovascular disease. Smoking, obesity, and prevalent longstanding diseases explained part of the inverse association between socioeconomic status and the inflammation markers, but also other factors are involved. These factors may include prevalent or sub-clinical diseases, and behavioural and environmental factors, such as diet, exercise, and exposure to toxic substances at work or elsewhere, which were not included in our analyses. Birth weight, which is related to mothers' socioeconomic status, has been shown to predict the risk of cardiovascular and other lonstanding diseases later in life. ${ }^{43}$ Some studies have also shown an inverse association between birth weight and fibrinogen. ${ }^{44}$

The association between low level inflammation and cardiovascular risk is, even though already well established, quite a recent finding. ${ }^{2}$ Despite active research, it is not known yet, whether the risk of cardiovascular disease could be reduced by reducing the levels of inflammation markers. However, proper and equal prevention and treatment of longstanding conditions, such as dental and respiratory infections, may reduce the levels of inflammatory factors and the risk of cardiovascular disease among the people with low socioeconomic status.

In conclusion, sensitive markers of systemic inflammation had an inverse association with socioeconomic status. The inverse association was particularly strong among men below 60 years of age. Thus, systemic inflammation is a potential mediator, especially among young and middle aged men, for the association between socioeconomic status and cardiovascular disease. Prospective observational studies are needed to assess the quantitative importance of inflammation in the association between socioeconomic status and cardiovascular disease risk.

\section{Authors' affiliations}

P Jousilahti, V Salomaa, National Public Health Institute, Department of Epidemiology and Health Promotion, Helsinki, Finland

V Rasi, E Vahtera, Finnish Red Cross Blood Transfusion Service, Department of Haemostasis, Helsinki, Finland

T Palosuo, National Public Health Institute, Department of Health and

Disability, Laboratory of Immunobiology, Helsinki, Finland

Funding: this study was supported by the Finnish Academy (grant number 37808).

Conflicts of interest: none declared.

\section{REFERENCES}

1 Danesh J, Collins R, Peto R. Chronic infections and coronary heart disease: is there a link? Lancet 1997;350:430-6.

2 Ross R. Atherosclerosis - an inflammatory disease. N Engl J Med 1999;340:115-26.

3 Nieminen MS, Mattila K, Valtonen V. Infection and inflammation as risk factors for myocardial infarction. Eur Heart J 1993 (suppl K);14:12-16.

4 Tracy RP. Inflammation in cardiovascular disease. Circulation 1998;97:2000-2.

5 Kovanen PT, Mänttäri M, Palosuo T, et al. Prediction of myocardial infarction in dyslipidemic men by elevated levels of immunoglobulin classes A, E, and G, but not M. Arch Intern Med 1998;158:1434-9.

6 Libby P, Hansson GK. Involvement of the immune system in human atherogenesis: current knowledge and unanswered questions. Lab Invest $1991 ; 64: 5-15$

7 Saikku P, Leinonen M, Mattila K, et al. Serological evidence of an association of novel Chlamydia, TWAR, with chronic coronary artery disease and acute myocardial infarction. Lancet 1988;ii:983-6.

8 Patel P, Mendall MA, Carrington D, et al. Associations of Helicobacter pylori and Chlamydia pneumoniae infections with coronary heart disease and cardiovascular risk factors. BM 1995;311:711-14.

9 Mattila KJ, Nieminen MS, Valtonen VV, et al. Association between dental health and acute myocardial infarction. BM 1989;298:779-81

10 McDonald K, Rector TS, Braulin EA, et al. Cytomegalovirus disease link to coronary artery disease. Am J Cardiol 1989;64:359-62.

11 Roivainen $M$, Alfthan $G$, Jousilahti $P$, et al. Enterovirus infections as a possible risk factor for myocardial infarction. Circulation 1998;98:2534-7.

12 Nieto FJ, Sorlie P, Comstock GW, et al. Cytomegalovirus infection, lipoprotein (a), and hypercoagulability: an atherogenic link. Arterioscler Throm Vasc Biol 1997;17:1780-5.
13 Koenig W Sund $M$, Fröhlich $M$, et al. C-reactive protein, a sensitive marker of inflammation, predicts future risk of coronary heart disease in initially healthy middle-aged men. Results from the MONICA (Monitoring trends and determinants in cardiovascular disease) Augsburg Cohort study, 1984 to 1992. Circulation 1999:99:237-42.

14 Kuller LH, Tracy RP, Shaten J, et al. (for the MRFIT Research Group). Relation of C-reactive protein and coronary heart disease in the MRFIT nested case-control study. Am J Epidemiol 1996;144:537-47

15 Ernst E, Resch K. Fibrinogen as cardiovascular risk factor: a meta-analysis and review of the literature. Ann Intern Med 1993; 118:956-63.

16 Thompson SG, Kienast J, Pyke SDM, et al. Hemostatic factors and risk of myocardial infarction or sudden death in patients with angina pectoris: European Concerted Action on Thrombosis and Disabilities Angina Pectoris Study Group. N Engl J Med 1995;332:635-41.

17 Malle E, Steinmetz A, Raynes JG. Serum amyloid A (SAA): an acute phase protein and apolipoprotein. Atherosclerosis 1993;102:131-46.

18 Kisilevsky R, Lindhorst E, Ancsin JB, et al. Acute phase serum amyloid A (SAA) and cholesterol transport during acute inflammation: a hypothesis. Int J Exp Clin Invest 1996;3:252-60.

19 Jousilahti $\mathbf{P}$, Salomaa V, Rasi V, et al. The association of C-reactive protein, serum amyloid a and fibrinogen with prevalent coronary heart disease-baseline findings of the PAIS project. Atherosclerosis 2000; 156:451-6.

20 Valkonen $\mathbf{T}$. Trends in regional and socio-economic mortality differentials in Finland. Int J Health Sci 1992;3:157-66.

21 Kunst AE, Mackenbach JP. The size of mortality differences associated with educational level in nine industrialized countries. Am J Public Health 1994;84:932-7.

22 Kunst AE, Mackenbach JP. International variation in the size of mortality differences associated with occupational status. Int J Epidemiol 1994;23:742-50.

23 Wilson TW, Kaplan GA, Kauhanen J, et al. Association between plasma fibrinogen concentration and five socioeconomic indices in the Kuopio Ischemic Heart Disease Risk Factor Study. Am J Epidemiol 1993; 137:292-300.

24 Rimm EB, Stampfer M, Giovannucci E, et al. Body size and fat distribution as predictors of coronary heart disease among middle-aged and older US men. Am J Epidemiol 1995;141:1117-27.

25 Clauss A. Gerinnungsphysiologische Schnell Methode zur Bestimmung des Fibrinogens. Acta Haemal 1957; 17:237-46.

26 Lagrand WK, Visser CA, Hermens WT, et al. C-reactive protein as a cardiovascular risk factor. More than an epiphenomenon? Circulation 1999:100:96-102.

27 Berk BC, Weintraub WS, Alexander RW. Elevation of C-reactive protein in "active" coronary artery disease. Am J Cardiol 1990;65: 168-72.

28 Mendall MA, Patel P, Ballam L, et al. C-reactive protein and its relation to cardiovascular risk factors: a population-based cross-sectional study. BM 1996;312:1061-5.

29 Tracy RP, Lemaitre RN, Psaty BM, et al. Relationship of C-reactive protein to risk of cardiovascular disease in the elderly. Arterioscler Thromb Vasc Biol 1997;17:1121-7.

30 Langrand WK, Niessen HW, Wolbink GJ, et al. C-reactive protein colocalizes with complement in human hearts during acute myocardial infarction. Circulation 1997;95:97-103.

31 Ridker PM, Glynn RJ, Hennekens CH. C-reactive protein adds to the predictive value of total and $\mathrm{HDL}$ cholesterol in determining risk of firs myocardial infarction. Circulation 1998;97:2007-11.

32 Ridker PM, Hennekens $\mathrm{CH}$, Buring JE, et al. C-reactive protein and other markers of inflammation in the prediction of cardiovascular disease in women. N Engl J Med 2000;342:836-43.

33 Fyfe AL, Rothenberg LS, DeBeer FC, et al. Association between serum amyloid A proteins and coronary artery disease: evidence from two distinct arteriosclerotic process. Circulation 1997;96:2914-19.

34 Pekkanen J, Tuomilehto J, Uutela A, et al. Social class, health behaviour, and mortality among men and women in eastern Finland. BM 1995;311:589-93.

35 Yudkin JS, Stehouwer CDA, Emeis JJ, et al. C-reactive protein in healthy subjects: Associations with obesity, insulin resistance, and endothelial dysfunction. A potential role for cytokines originating from adipose tissue? Arterioscler Thromb Vasc Biol 1999;19:972-8.

36 Kannel WB, Wolf PA, Castelli WP, et al. Fibrinogen and risk of cardiovascular disease. The Framingham Study. JAMA 1987;258: $1183-6$.

37 Ernst E. The Role of fibrinogen as cardiovascular risk factor. Atherosclerosis 1993;100:1-12.

38 Mendall MA, Patel P, Asante M, et al. Relation of serum cytokine concentrations to cardiovascular risk factors and coronary heart disease Heart 1997;78:273-7.

39 Feghali CA, Wright TM. Cytokines and acute and chronic inflammation. Front Biosci 1997;2:12-26.

40 Cohem MC, Cohen S. Cytokine function: a study in biologic diversity. Am J Clin Pathol 1996; 105:589-98

41 Danesh J, Muir J, Wong YK, et al. Risk factors for coronary heart disease and acute-phase proteins. A population-based study. Eur Heart J 1999:20:954-9.

42 Iso $\mathbf{H}$, Shimamoto $T$, Sato $T$, et al. Passive smoking and plasma fibrinogen concentrations. Am J Epidemiol 1996;144:1 151-4.

43 Barker DJ, Gluckman PD, Godfrey KM, et al. Fetal nutrition and cardiovascular disease in adult life. Lancet 1993;341:938-41.

44 Martyn CN, Meade TW, Stirling Y, et al. Plasma concentrations of fibrinogen and factor VII in adult life and their relation to intra-uterin growth. Br J Haematol 1995;89: 142-6. 